\title{
Estradiol-enhanced osteogenesis of rat bone marrow stromal cells is associated with the JNK pathway
}

\author{
NAN SONG ${ }^{1}$, ZHI-MIN WANG ${ }^{2}$, LI-JUAN HE ${ }^{3}$, YAN XU $^{4}$ and YAN-LING REN ${ }^{3}$ \\ ${ }^{1}$ Key Laboratory of Ministry of Education for TCM Viscera-State Theory and Applications, Ministry of Education of China; \\ ${ }^{2}$ The Graduate School; ${ }^{3}$ School of Chinese Medical Formulae, College of Basic Medicine; ${ }^{4}$ College of Pharmacy, \\ Liaoning University of Traditional Chinese Medicine, Shenyang, Liaoning 110847, P.R. China
}

Received December 29, 2015; Accepted January 5, 2017

DOI: $10.3892 / \mathrm{mmr} .2017 .7699$

\begin{abstract}
Bone marrow stromal cells (BMSCs) can differentiate into osteoblasts. The present study investigated the osteogenic effects of estradiol, as well as the role of the c-Jun $\mathrm{N}$-terminal kinase (JNK) signaling pathway in promoting estradiol-enhanced osteogenesis of rat (r)BMSCs. rBMSCs were treated for 7 days with or without estradiol and further treated with or without the JNK-specific inhibitor SP600125. The role of estrogen during rBMSC osteogenesis was evaluated by alkaline phosphatase activity and mineralized nodule formation using the Gomori method and Alizarin red $\mathrm{S}$ staining, respectively. Subsequently, the mRNA expression levels of transforming growth factor- $\beta 1$ (TGF- $\beta 1$ ) and core-binding factor $\alpha 1(\mathrm{Cbf} \alpha 1)$ were evaluated by reverse transcription-quantitative polymerase chain reaction, and TGF- $\beta 1$, Cbf $\alpha 1$ and phosphorylated (p)-JNK protein expression was detected by western blotting. All groups treated with SP600125 expressed low levels of TGF- $\beta 1$ and Cbf $\alpha 1$ mRNA and protein, and low p-JNK protein expression. Compared with the control cells, rBMSCs cultured with estradiol exhibited a significant upregulation in the expression levels of osteogenic genes and proteins. The present study demonstrated that estradiol enhanced osteogenic differentiation of rBMSCs and that the JNK signaling pathway was involved in this process, providing insights into the molecular mechanisms involved in rBMSC osteogenesis upon estradiol stimulation.
\end{abstract}

\section{Introduction}

Osteoporosis is a very common disease in postmenopausal women. It is frequently associated with bone fractures, which

Correspondence to: Dr Yan-Ling Ren, School of Chinese Medical Formulae, College of Basic Medicine, Liaoning University of Traditional Chinese Medicine, 79 East Chongshan Road, Shenyang, Liaoning 110847, P.R. China

E-mail: renyanling1963@hotmail.com

Key words: estradiol, bone marrow stromal cells, osteogenesis, JNK signaling pathway, TGF- $\beta 1 /$ Smad signaling pathway are a major health problem worldwide (1). Osteoporosis is characterized by a reduction in bone mass caused by a disturbance in the ratio between bone formation and bone resorption. This imbalance may be associated with low blood serum estrogen levels in postmenopausal women (2). Estrogen deficiency is recognized as an important risk factor in the development of postmenopausal osteoporosis (3). Although prolonged exposure to relatively high doses of estrogen may result in sustained stimulation of osteoblastic activity in postmenopausal women (4), the precise mechanism by which estrogen enhances bone formation is not clear.

Osteoblast precursors are considered to be derived from marrow mesenchymal stem cells (MSCs) (5). It has been hypothesized that estrogen increases bone formation by enhancing the proliferation and differentiation of MSCs. Primary bone marrow stromal cells (BMSCs) are multipotent and capable of differentiating along the osteoblastic cell lineage (6). Rat BMSCs (rBMSCs) have been used in previous tissue engineering studies because they are strongly adherent, highly proliferative and readily available $(7,8)$. Therefore, rBMSCs were used in the present study to examine the contribution of estrogen to osteogenic differentiation.

Mitogen-activated protein kinases (MAPKs) are involved in the regulation of gene expression, mitosis, metabolism, motility, survival, apoptosis and differentiation of stem cells. MAPK subfamilies mediate various biological effects: Extracellular signal-regulated kinase 1 (ERK1) and ERK2 regulate cell differentiation and proliferation; c-Jun N-terminal kinases (JNKs) have an important role in cell cycle regulation, apoptosis and cellular stress; and p38 MAPKs affect numerous biological processes, such as proliferation, differentiation and survival, and responses to stress and inflammation in certain cell types (9). A number of previous studies have focused on the functions of ERK1/2 and p38 MAPKs in other contexts, such as tumor and diabetes studies $(10,11)$. MAPKs may also be activated during osteogenesis. The JNK signaling pathway has previously been demonstrated to serve a role in the regulation of osteogenic differentiation of MSCs (12). The present study hypothesized that estradiol may enhance the induction of osteogenesis by upregulating the JNK signaling pathway. To validate this hypothesis the underlying mechanisms of estradiol-induced osteogenesis in rBMSCs were investigated. 


\section{Materials and methods}

Ethics statement. A total of 5 male Sprague-Dawley rats (age, 6 weeks; weight $200 \pm 10 \mathrm{~g}$ ) were purchased from Vital River Laboratories Co., Ltd. (Beijing, China). Rats were housed in a climate-controlled environment (temperature, $22 \pm 1^{\circ} \mathrm{C}$; humidity, $50 \pm 5 \%$ ) with a $12 / 12 \mathrm{~h} \mathrm{light/dark}$ cycle and ad libitum access to food and water. All procedures were performed under $10 \%$ chloral hydrate anesthesia when necessary. The rats were sacrificed by asphyxiation with $\mathrm{CO}_{2}$. All experimental procedures were approved by (permit no. 2011-167) and followed the guidelines of the Animal Ethics Committee of Liaoning University of Traditional Chinese Medicine (Shenyang, China).

Primary culture of rBMSCs. rBMSCs were isolated from the bone marrow of the posterior tibias and femurs of 8-week-old Sprague-Dawley rats. Bone marrow cells were flushed out with $\alpha$-minimum essential medium ( $\alpha$-MEM; HyClone; GE Healthcare Life Sciences, Logan, UT, USA) and centrifuged at $300 \mathrm{x}$ g for $3 \mathrm{~min}$. rBMSCs adhering to the plastic surface were selected for further experimentation. Primary cells $\left(1 \times 10^{7}\right.$ cells $\left./ \mathrm{ml}\right)$ were cultured with $\alpha$-MEM containing 10\% FBS (HyClone; GE Healthcare Life Sciences) for 5 days $\left(37^{\circ} \mathrm{C}, 5 \% \mathrm{CO}_{2}\right)$, changing the medium every 3 days. At $80 \%$ confluence, the cells were passaged at a ratio of 1:2. All experiments were performed using cells cultured to the fourth passage.

Flow cytometric analysis. Cells were grown to passage 4, washed twice with PBS and incubated with $0.25 \%$ trypsin for $\sim 1 \mathrm{~min}$; the reaction was stopped by adding PBS. Trypsinized cells were transferred to tubes at a density of $1 \times 10^{6}$ cells $/ \mathrm{ml}$. The cells were then stained with antibodies (1:200) against cell surface markers CD29 (cat. no. 102215), CD90.1 (cat. no. 202515) and CD45 (cat. no. 202207) (BioLegend, Inc., San Diego, CA, USA) in the dark for $30 \mathrm{~min}$ at $4^{\circ} \mathrm{C}$, followed by centrifugation at $300 \mathrm{x}$ g for $5 \mathrm{~min}$. Following centrifugation, cells were washed with PBS $(1 \mathrm{ml})$, centrifuged at $300 \mathrm{x} \mathrm{g}$ for $5 \mathrm{~min}$ and resuspend in $300 \mu \mathrm{l}$ PBS. Cells were analyzed using a BD FACSCalibur flow cytometer (BD Biosciences, San Jose, CA, USA), and the FlowJo software (FlowJo, LLC, Ashland, OR, USA).

Alkaline phosphatase (ALP) activity assay. A colorimetric p-nitrophenyl phosphate (pNPP) assay (Sigma-Aldrich; Merck KGaA, Darmstadt, Germany) was used to evaluate ALP activity and to quantify the osteogenesis of rBMSCs cultured in either induction medium (IND group; $10^{-7} \mathrm{~mol}$ dexamethasone, $50 \mu \mathrm{mol}$ ascorbic acid and $10 \mathrm{mmol} / \mathrm{l}$ sodium $\beta$-glycerophosphate) or induction medium supplemented with estradiol (E2 group) at concentrations of $10^{-6}$, $10^{-7}, 10^{-8}$ and $10^{-9} \mathrm{~mol}$. ALP activity was assayed at days 5,7 and 9. Briefly, rBMSCs $\left(5 \times 10^{4}\right.$ cells $)$ were diluted in $0.2 \mathrm{ml}$ of control ( $\alpha$-MEM supplemented with 10\% FBS), IND or E2 medium and transferred to a 96 -well plate (8 wells/experimental group). Triton X-100 (100 $\mu 1 ; 0.1 \%$ Triton X-100) was then added and the cells were incubated at $-20^{\circ} \mathrm{C}$ overnight. Following overnight incubation, cells were lysed in $100 \mu \mathrm{l}$ $\mathrm{pNPP} /$ diethanolamine buffer $(1: 1)$ at $37^{\circ} \mathrm{C}$ for $30 \mathrm{~min}$. The reaction was terminated by adding $80 \mu \mathrm{l} \mathrm{NaOH}$ buffer $(0.5 \mathrm{~mol})$. All wells were analyzed in a microplate reader at an optical density of $415 \mathrm{~nm}$.

ALP staining assay. The ALP staining assay comprised 3 experimental groups: i) A control group, which consisted of rBMSCs cultured in growth medium (10\% FBS in $\alpha$-MEM); ii) an IND group; and iii) an E2 group cultured in IND + estradiol $\left(10^{-8} \mathrm{~mol}\right)$. Each group was further divided into 2 treatment groups in which the medium was supplemented with or without the JNK-specific inhibitor SP600125 $\left(10 \mu \mathrm{mol}, 37^{\circ} \mathrm{C}, 30 \mathrm{~min}\right)$ (Merck KGaA). The 6 experimental groups were subjected to ALP staining assays using the Gomori method. Briefly, cells were seeded at a density of $1 \times 10^{5}$ cells/well in 24-well plates with 3 wells/group. Following incubation for 14 days at $37^{\circ} \mathrm{C}$, the cells were stained with fresh ALP incubation buffer (2\% barbitone sodium, $2 \%$ anhydrous calcium chloride, $3 \%$ $\beta$-sodium glycerol-phosphate, $2 \%$ magnesium sulfate and $5 \mathrm{ml}$ distilled water, $\mathrm{pH} 9.4$ ) for $4 \mathrm{~h}$ at $37^{\circ} \mathrm{C}$. The cells were rinsed with ultrapure water for $10 \mathrm{~min}$, treated with $2 \%$ cobalt nitrate for $5 \mathrm{~min}$, followed by $1 \%$ ammonium sulfide solution for $1 \mathrm{~min}$. After drying, the stained cells were imaged under a light microscope and quantitative analysis was conducted using the BI-2000 digital image analysis system (Chengdu TME Technology Co., Ltd., Chengdu, China).

Alizarin red S staining. Alizarin red S staining was used to examine the mineralized nodule formation of rBMSCs. Cells were prepared as described for the ALP staining assay and cultured for 21 days. The cells were stained with alizarin red $\mathrm{S}$ $[0.1 \%(w / v)$ in Tris- $\mathrm{HCl}]$ for $2 \mathrm{~h}$ at $37^{\circ} \mathrm{C}$, then washed three times with PBS. Finally, mineralized nodules were imaged under an inverted light microscope and quantitative analysis was conducted using the BI-2000 digital image analysis system (Chengdu TME Technology Co., Ltd.).

Reverse transcription-quantitative polymerase chain reaction (RT-qPCR). RT-qPCR analysis was performed on the 6 experimental groups described in the aforementioned ALP staining assay. Cells $\left(3 \times 10^{5}\right)$ were seeded in flasks and cultured with the relevant incubation medium (control, IND or E2) with or without $10 \mu \mathrm{mol} \mathrm{SP600125} \mathrm{for} 7$ days. Total RNA was extracted with TRIzol reagent (Invitrogen; Thermo Fisher Scientific, Inc., Waltham, MA, USA), and quantified with a spectrophotometer at 260/280 nm. Following the removal of genomic DNA using gDNA Eraser (Takara Biotechnology Co., Ltd., Dalian, China), total RNA was reverse transcribed using a PrimeScript First Strand cDNA Synthesis kit (Takara Biotechnology Co., Ltd.) according to the manufacturer's instructions.

PCR was performed using a SYBR Green kit (Takara Biotechnology Co., Ltd.) and an Mx3000P qPCR system (Agilent Technologies, Inc., Santa Clara, CA, USA) using the following cycling conditions: 1 cycle of $95^{\circ} \mathrm{C}$ for $2 \mathrm{~min}$, followed by 40 cycles of $95^{\circ} \mathrm{C}$ for $30 \mathrm{sec}$ and $60^{\circ} \mathrm{C}$ for $30 \mathrm{sec}$. Primers were designed using Primer Premier 5.0 (Premier Biosoft International, Palo Alto, CA, USA) and were custom-made by Takara Biotechnology Co., Ltd. Gene expression was normalized relative to that of the housekeeping gene GAPDH. The following rat primers were used: GAPDH forward, 
5'-TGTGTCCGTCGTGGATCTGA-3' and reverse, 5'-TTGCTG TTGAAGTCGCAGGAG-3'; core-binding factor $\alpha 1$ (Cbf $\alpha 1$ ) forward, 5'-CACTGGCGGTGCAACAAGA-3' and reverse, 5'-ATGACGGTAACCACAGTCCCATC-3'; and transforming growth factor- $\beta 1$ (TGF- $\beta 1$ ) forward, 5 -GTGTGGAGCAAC ATGTGGAACTCTA-3' and reverse, 5'-CGCTGAATCGAA AGCCCTGTA-3'. Relative mRNA expression levels were quantified using the $2^{-\Delta \Delta C q}$ method (13).

Western blot analysis. rBMSCs were prepared as described in the ALP staining assay and proteins were extracted using a Mammal Tissue Protein Extraction kit (Boster Biological Technology, Pleasanton, CA, USA) with 1 mmol phenylmethanesulfonyl fluoride. Protein quantification was performed with a bicinchoninic acid protein assay. Protein samples $(50 \mu \mathrm{g})$ were separated by $12 \%$ SDS-PAGE, transferred to a polyvinylidene difluoride membrane, and incubated with primary and secondary antibodies $\left(37^{\circ} \mathrm{C} ; 1 \mathrm{~h}\right)$. The primary antibodies were against GAPDH (cat. no. 5174), total JNK (cat. no. 9252), phosphorylated (p)-JNK (cat. no. 9251) (Cell Signaling Technology, Inc., Danvers, MA, USA), TGF- $\beta 1$ (cat. no. sc-146; Santa Cruz Biotechnology, Inc., Dallas, TX, USA) and Cbf $\alpha 1$ (cat. no. ab76956; Abcam, Cambridge, UK). Protein bands were visualized using an enhanced chemiluminescence kit (Thermo Fisher Scientific, Inc.) and exposed to X-ray films (Kodak, Rochester, NY, USA). Densitometric analysis was performed with AlphaView SA Software (ProteinSimple; Bio-Techne, Minneapolis, MN, USA) to analyze protein quantification. And the results were expressed as a ratio of the level of expression of the target protein to GAPDH expression. The level of JNK phosphorylation was represented by the ratio of p-JNK to JNK protein expression levels.

Statistical analysis. Results are presented as the mean \pm standard deviation. Multiple comparisons were performed by one-way analysis of variance followed by Fisher's least significant difference test using GraphPad Prism 5 (GraphPad Software, Inc., La Jolla, CA, USA). $\mathrm{P}<0.05$ was considered to indicate a statistically significant difference. Each experiment was repeated at least 3 times.

\section{Results}

Morphological features and characterization of rBMSCs by flow cytometry. rBMSCs were imaged under an inverted microscope (Fig. 1A). Primary cells were circular or oval in shape. Cells were passaged when they reached $80 \%$ confluence at 8-10 days incubation. Cells at passage 4 were larger and spindle-shaped (Fig. 1B). Cells were allowed to grow to $80 \%$ confluence in a culture flask.

Cells were harvested and subjected to flow cytometry to examine cell surface markers. A total of 97.72 and $84.71 \%$ of cells were positive for the mesenchymal surface markers CD29 and CD90.1, respectively (Fig. 1C and D); whereas $2.81 \%$ were positive for the hematopoietic stem cell marker CD45 (Fig. 1E).

rBMSC ALP activity. ALP is an enzyme that is expressed during rBMSC osteogenesis and is considered a well-defined
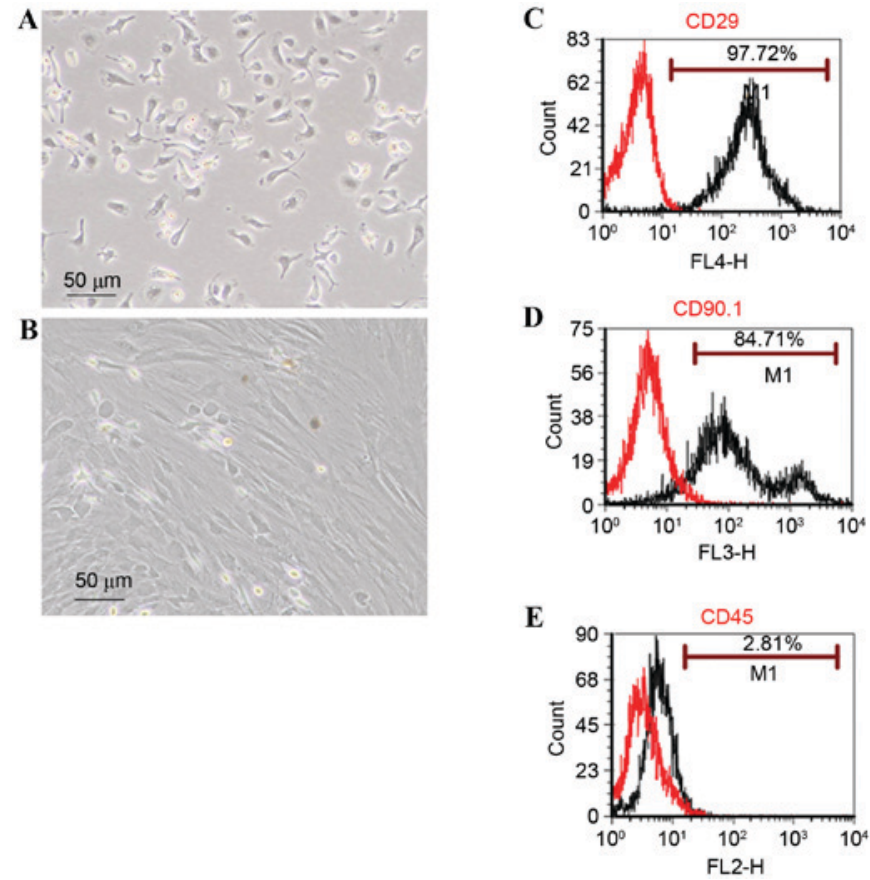

Figure 1. Morphology and cell surface markers of rBMSCs. Morphology of primary rBMSCs at (A) 5 days and (B) passage 4 rBMSCs at 6 days (magnification, x100). Flow cytometric analysis of rBMSCs for (C) CD29, (D) CD90.1 and (E) CD45. rBMSCs, rat bone marrow stromal cells.

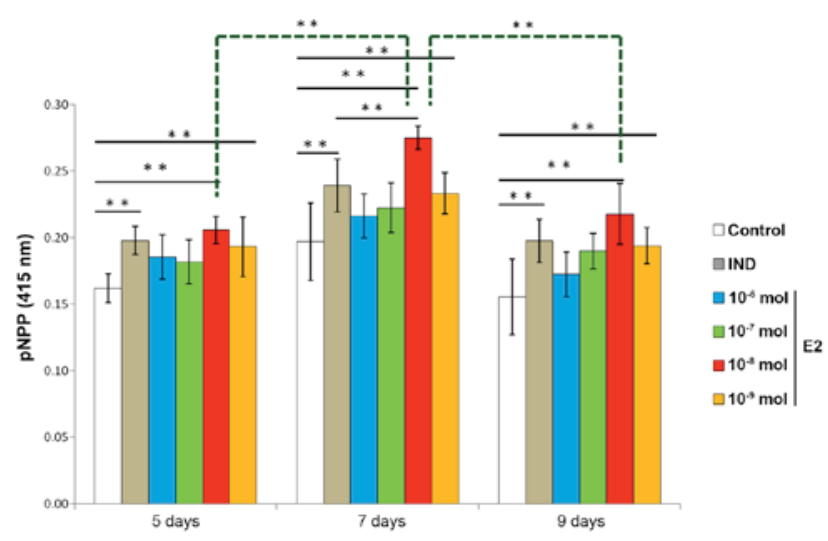

Figure 2. ALP activity of rBMSCs treated with various concentrations of E2. The optimal E2 concentration and incubation time were determined as $10^{-8} \mathrm{~mol}$ and 7 days, respectively ( $\left.{ }^{* *} \mathrm{P}<0.01\right)$. E2, estradiol; IND, induction medium; pNPP, p-nitrophenyl phosphate; rBMSCs, rat bone marrow stromal cells.

and early marker for their differentiation. ALP activity was examined to evaluate the optimal concentration and time required for estradiol-induced rBMSC osteogenesis. Compared with the control group, two of the E2 groups $\left(10^{-8} \mathrm{~mol}\right.$ and $10^{-9}$ mol estradiol) and the IND group exhibited higher levels of ALP on days 5, 7 and 9. Compared with the IND group, the $10^{-8}$ mol E2 group had significantly higher levels of ALP on day $7(\mathrm{P}<0.01)$; rBMSCs treated with $10^{-8}$ mol estradiol also had significantly higher levels of ALP on day 7 than those cells receiving the same treatment on days 5 and $9(\mathrm{P}<0.01)$. Therefore, estradiol induction of osteogenesis was considered optimal at a concentration of $10^{-8}$ mol estradiol and 7 days incubation (Fig. 2). 
A

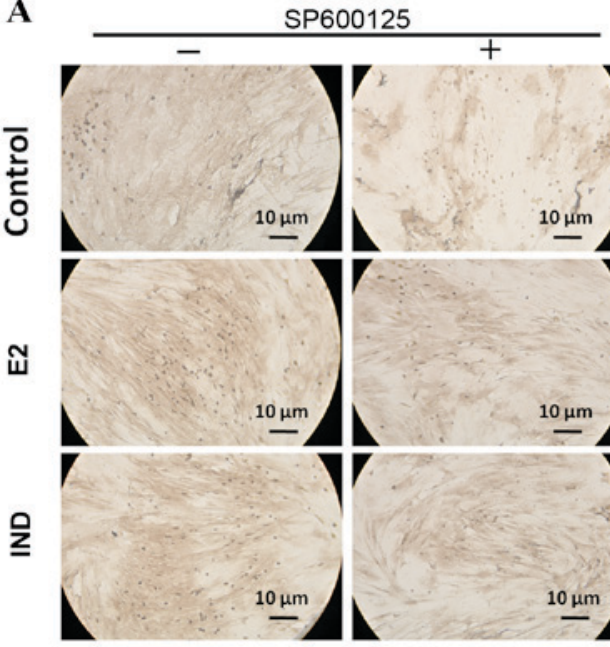

B

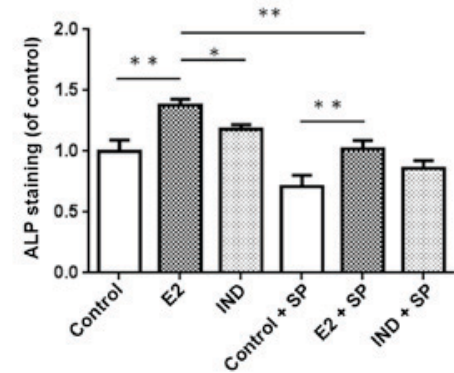

C

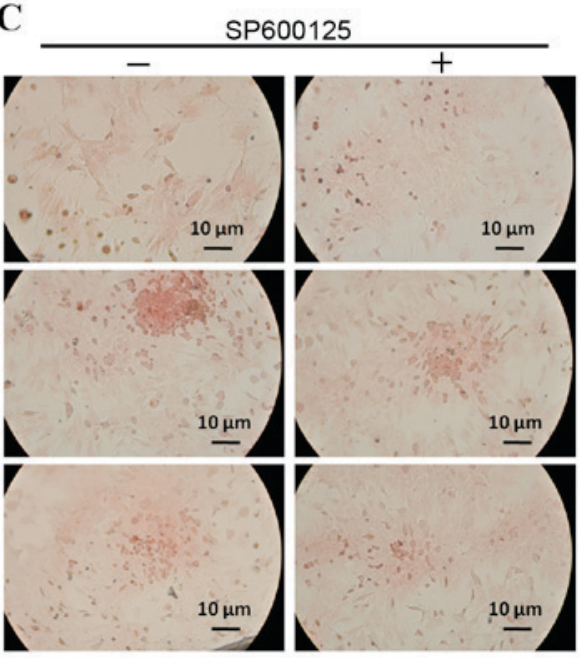

D

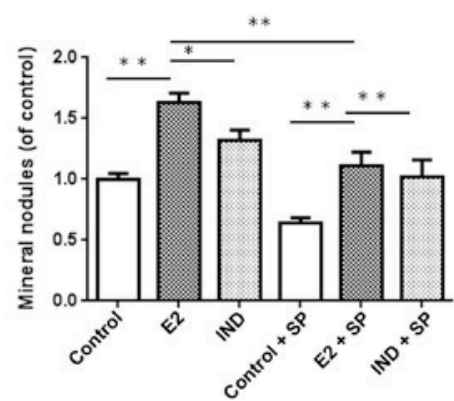

Figure 3. ALP staining and mineral nodule formation of rat bone marrow stromal cells. (A and B) ALP staining was performed using Gomori's method. (C and D) Mineral nodule formation was determined by Alizarin red S staining. $\mathrm{P}<0.05$ and ${ }^{* *} \mathrm{P}<0.01$. ALP, alkaline phosphatase; E2, estradiol; IND, induction medium; SP, SP600125.

ALP staining and mineral nodule formation. Cell cultures were imaged with an inverted light microscope (Fig. 3). The E2 and IND rBMSC groups had significantly higher levels of ALP compared with the control group $(\mathrm{P}<0.01)$ (Fig. 3A and B). The level of ALP in rBMSCs of the E2 group was significantly higher compared with cells in the IND group $(\mathrm{P}<0.05)$.

Alizarin red $\mathrm{S}$ staining was used to examine the formation of mineralized nodules in the various rBMSC cultures. As shown in Fig. 3C and D, the number of nodules forming in the E2 and IND groups were significantly larger compared with the control group, and those of the E2 group were significantly larger compared with the IND group at 21 days $(\mathrm{P}<0.01$ or $\mathrm{P}<0.05)$. Treatment with the JNK inhibitor SP600125 reduced the osteogenesis-stimulating effects of estradiol; however, nodule formation remained significantly higher in the E2 and IND groups, compared with the control group.

JNK phosphorylation. The relative level of p-JNK compared with the total JNK protein expression in rBMSCs was assayed by western blotting (Fig. 4). Compared with the E2 group, the control and IND groups had lower levels of p-JNK $(\mathrm{P}<0.01)$. Addition of the JNK inhibitor SP600125 significantly reduced the levels of JNK and p-JNK protein expression under all conditions. However, the expression levels of p-JNK in the E2 and IND groups co-cultured with SP600125 remained higher compared with the control group treated with the inhibitor $(\mathrm{P}<0.05)$.
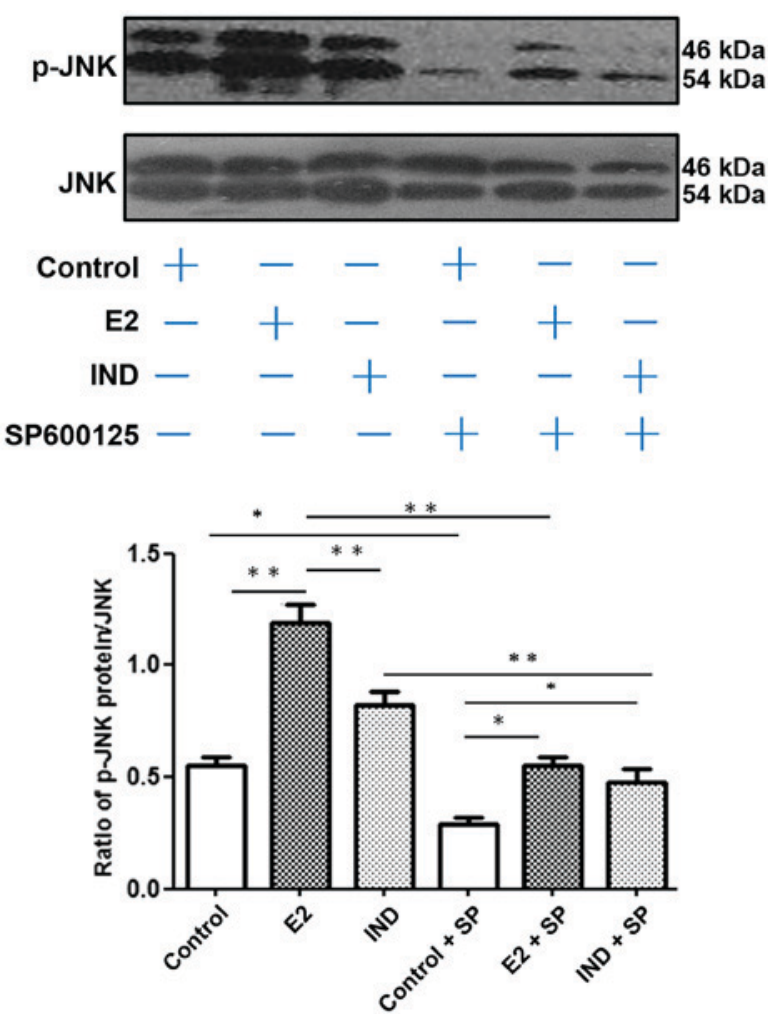

Figure 4. Western blot analysis of JNK and p-JNK protein in rat bone marrow stromal cells. Significant differences among each group are noted by ${ }^{*} \mathrm{P}<0.05$ and ${ }^{* *} \mathrm{P}<0.01$. E2, estradiol; IND, induction medium; JNK, c-Jun N-terminal kinase; p, phosphorylated; SP, SP600125. 

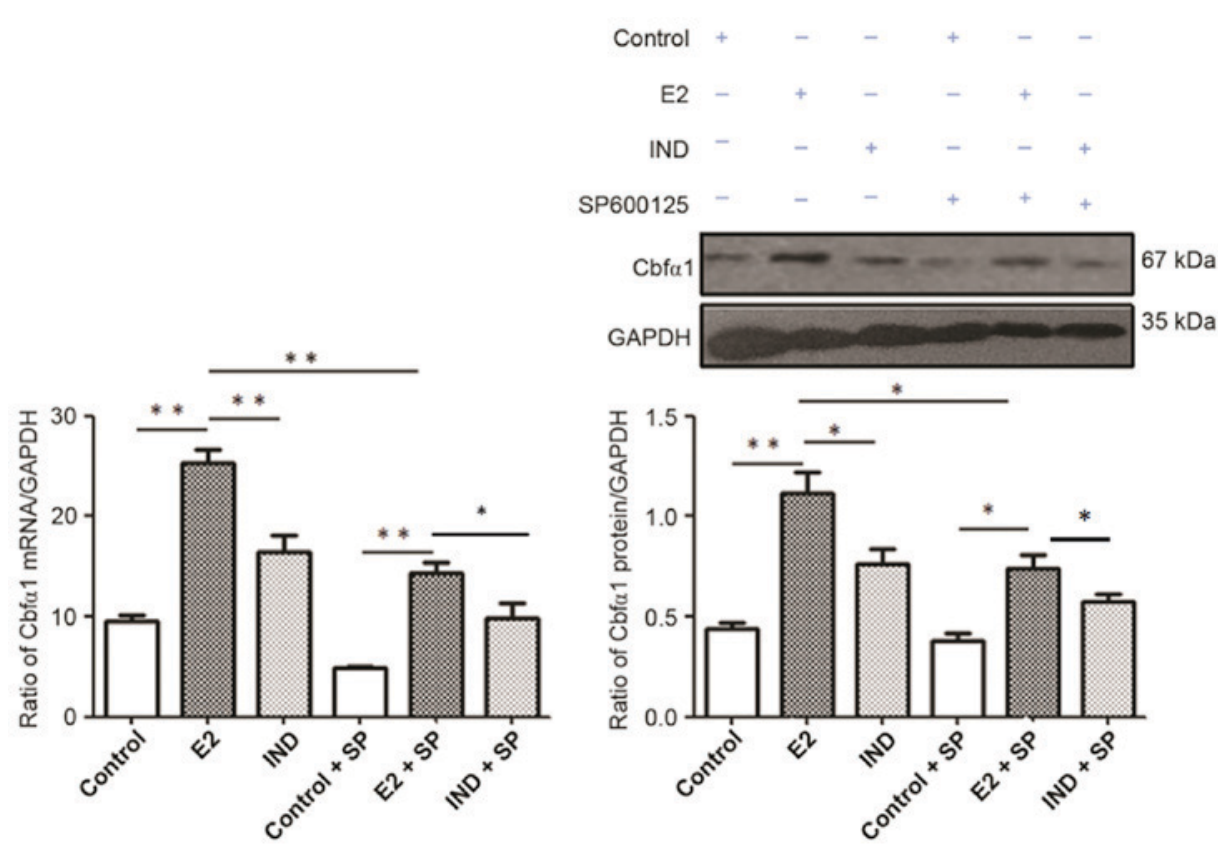

Figure 5. Cbf $\alpha 1$ mRNA and protein expression levels in rat bone marrow stromal cells. Reverse transcription-quantitative polymerase chain reaction and western blot analysis of Cbf $\alpha 1$ mRNA and protein expression, respectively. GAPDH blot is the same as in Fig. 6, as Cbf $\alpha 1$, TGF- $\beta 1$ and GAPDH were all probed for on the same membrane. ${ }^{*} \mathrm{P}<0.05$ and ${ }^{* *} \mathrm{P}<0.01$. Cbf $\alpha 1$, core-binding factor $\alpha 1$; E2, estradiol-treated group; IND, induction medium group; SP, SP600125.
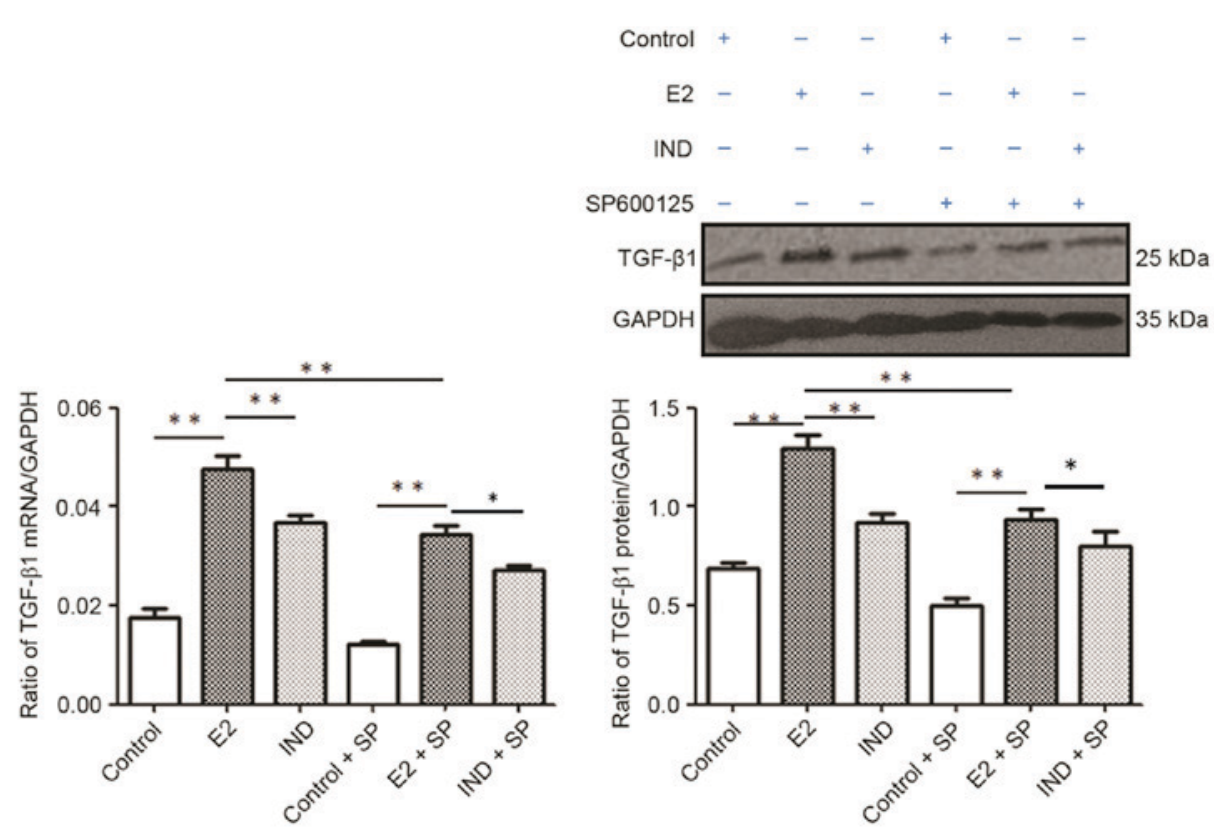

Figure 6. TGF- $\beta 1$ mRNA and protein expression levels in rat bone marrow stromal cells. Reverse transcription-quantitative polymerase chain reaction and western blot analyses were used to detect TGF- $\beta 1 \mathrm{mRNA}$ and protein levels, respectively. GAPDH blot is the same as in Fig. 5, as Cbf $\alpha 1$, TGF- $\beta 1$ and GAPDH were all probed for on the same membrane. ${ }^{*} \mathrm{P}<0.05$ and ${ }^{* *} \mathrm{P}<0.01$. E2, estradiol group; IND, induction medium; SP, SP600125; TGF- $\beta 1$, transforming growth factor- $\beta 1$.

Cbfal $\mathrm{mRNA}$ and protein expression. Cbf $\alpha 1 \mathrm{mRNA}$ and protein expression levels were significantly higher in rBMSCs from the E2 group compared with the control and IND groups $(\mathrm{P}<0.01$ or $\mathrm{P}<0.05$; Fig. 5). Although the levels of Cbf $\alpha 1 \mathrm{mRNA}$ and protein expression were lower in all groups following the addition of SP600125, they remained higher in the E2 group compared with the IND and control groups $(\mathrm{P}<0.01$ or $\mathrm{P}<0.05)$.
TGF- $\beta 1 \mathrm{mRNA}$ and protein expression. The E2 group had significantly higher levels of TGF- $\beta 1$ mRNA and protein expression compared with the control and IND groups $(\mathrm{P}<0.01$; Fig. 6). The expression levels of TGF- $\beta 1 \mathrm{mRNA}$ and protein were subsequently lower in each group when co-treated with SP600125; in addition, the levels were significantly higher in the E2 group compared with the control or IND groups $(\mathrm{P}<0.01)$. 


\section{Discussion}

The present study investigated the effects of the estrogen steroid sex hormone estradiol on the osteogenic differentiation of rBMSCs. Estradiol treatment was associated with an increase in osteogenic induction, as determined by examining markers of osteogenesis, such as ALP activity and expression, and the formation of mineralized nodules. ALP is an early stage marker, whereas the formation of mineralized nodules is an indicator of middle- and late-stage osteogenesis $(14,15)$. The present study determined the optimal concentration of estradiol treatment in rBMSC culture was $10^{-8} \mathrm{~mol}$ and the optimal incubation time was 7 days. Results indicated that osteogenesis in rBMSCs treated with estradiol was more robust compared with those treated with IND media, as indicated by higher levels of ALP and stronger formation of mineralized nodules.

Animal models of osteoporosis, such as ovariectomized mice, are widely used to study the signaling cascades that control rBMSC osteogenesis, including research on postmenopausal osteoporosis (16). However, it remains unclear how estrogen contributes to the osteogenic differentiation of stem cells. A previous study demonstrated that the JNK signaling pathway is involved in the regulation of osteogenesis (12). Guicheux et al (17) reported that bone morphogenetic protein 2 regulated osteoblastic differentiation of stem cells through activation of the JNK and p38 MAPK signaling pathways. Therefore, the present study explored the role of JNK signaling in the estrogen-mediated regulation of osteogenic differentiation of rBMSCs. The results demonstrated that estradiol is associated with higher mRNA and protein expression levels of p-JNK and Cbf $\alpha 1$ in rBMSC cultures. Cbf $\alpha 1$ is a transcription factor that regulates the expression of numerous genes relevant to osteogenesis $(18,19)$. It is a major factor in the initiation and regulation of early osteogenesis, and serves a crucial role in the late mineralization stages (20). Notably, the present study revealed that the E2 group had relatively high levels of p-JNK and Cbf $\alpha 1$ mRNA and protein following treatment with the JNK-specific inhibitor SP600125, compared with cells in the control and IND groups. These data indicated that JNK signaling my serve a crucial role during estrogen-induced osteogenesis of rBMSCs.

Furthermore, results from the present study demonstrated that the mRNA and protein expression levels of TGF- $\beta 1$ were higher in the E2 group compared with the IND and control groups, and were decreased in the presence of SP600125. TGF- $\beta 1$ may stimulate the phosphorylation of JNK, which enables the signal to be transferred into the nucleus to regulate the osteogenic differentiation of rBMSCs. In conclusion, the present study provides evidence to suggest that estradiol may promote rBMSC osteogenesis by activating the JNK signaling pathway. These data support the hypothesis that estradiol-induced JNK signaling contributes to osteogenesis and the prevention of postmenopausal osteoporosis.

\section{Acknowledgements}

The present study was supported by the National Natural Science Foundation of China (grant nos. 81373527 and 81774185).

\section{References}

1. Siris ES, Miller PD, Barrett-Connor E, Faulkner KG, Wehren LE, Abbott TA, Berger ML, Santora AC and Sherwood LM: Identification and fracture outcomes of undiagnosed low bone mineral density in postmenopausal women: Results from the National Osteoporosis Risk Assessment. JAMA 286: 2815-2822, 2001.

2. Rachner TD, Khosla S and Hofbauer LC: Osteoporosis: Now and the future. Lancet 377: 1276-1287, 2011.

3. Al-Rahbi B, Zakaria R, Othman Z, Hassan A and Ahmad AH: Enhancement of BDNF concentration and restoration of the hypothalamic-pituitary-adrenal ax is accompany reduced depressive-like behaviour in stressed ovariectomised rats treated with either Tualang honey or estrogen. ScientificWorldJournal 2014: 310821, 2014.

4. Chen FP, Hu CH and Wang KC: Estrogen modulates osteogenic activity and estrogen receptor mRNA in mesenchymal stem cells of women. Climacteric 16: 154-160, 2013.

5. Prockop DJ: Marrow stromal cells as stem cells for nonhematopoietic tissues. Science 276: 71-74, 1997.

6. Tan SL, Ahmad TS, Selvaratnam L and Kamarul T: Isolation, characterization and the multi-lineage differentiation potential of rabbit bone marrow-derived mesenchymal stem cells. J Anat 222: 437-450, 2013.

7. Chen M, Feng W, Cao H, Zou L, Chen C, Baatrup A, Nielsen AB, Li H, Kassem M, Zou X and Bünger C: A traditional Chinese medicine formula extracts stimulate proliferation and inhibit mineralization of human mesenchymal stem cells in vitro. J Ethnopharmacol 125: 75-82, 2009.

8. Zhou DA, Zheng HX, Wang CW, Shi D and Li JJ: Influence of glucocorticoids on the osteogenic differentiation of rat bone marrow-derived mesenchymal stem cells. BMC Musculoskelet Disord 15: 239, 2014.

9. Cargnello $\mathrm{M}$ and Roux PP: Activation and function of the MAPKs and their substrates, the MAPK-activated protein kinases. Microbiol Mol Biol Rev 75: 50-83, 2011.

10. Kim HK, Kim MG and Leem KH: Osteogenic activity of collagen peptide via ERK/MAPK pathway mediated boosting of collagen synthesis and its therapeutic efficacy in osteoporotic bone by back-scattered electron imaging and microarchitecture analysis. Molecules 18: 15474-15489, 2013.

11. Sonowal H, Kumar A, Bhattacharyya J, Gogoi PK and Jaganathan BG: Inhibition of actin polymerization decreases osteogeneic differentiation of mesenchymal stem cells through p38 MAPK pathway. J Biomed Sci 20: 71, 2013.

12. Kwon HS, Johnson TV and Tomarev SI: Myocilin stimulates osteogenic differentiation of mesenchymal stem cells through mitogen-activated protein kinase signaling. J Biol Chem 288: 16882-16894, 2013.

13. Livak KJ and Schmittgen TD: Analysis of relative gene expression data using real-time quantitative PCR and the 2(-Delta Delta C(T)) method. Methods 25: 402-408, 2001.

14. Marom R, Shur I, Solomon R and Benayahu D: Characterization of adhesion and differentiation markers of osteogenic marrow stromal cells. J Cell Physiol 202: 41-48, 2005.

15. Li XD, Wang JS, Chang B, Chen B, Guo C, Hou GQ, Huang DY and Du SX: Panax notoginseng saponins promotes proliferation and osteogenic differentiation of rat bone marrow stromal cells. J Ethnopharmacol 134: 268-274, 2011.

16. Komori T: Animal models for osteoporosis. Eur J Pharmacol 759: 287-294, 2015.

17. Guicheux J, Lemonnier J, Ghayor C, Suzuki A, Palmer G and Caverzasio J: Activation of p38 mitogen-activated protein kinase and c-Jun-NH2-terminal kinase by BMP-2 and their implication in the stimulation of osteoblastic cell differentiation. J Bone Miner Res 18: 2060-2068, 2003.

18. Byers BA, Pavlath GK, Murphy TJ, Karsenty G and Garcia AJ: Cell-type-dependent up-regulation of in vitro mineralization after overexpression of the osteoblast-specific transcription factor Runx2/Cbfal. J Bone Miner Res 17: 1931-1944, 2002.

19. Teplyuk NM, Haupt LM, Ling L, Dombrowski C, Mun FK, Nathan SS, Lian JB, Stein JL, Stein GS, Cool SM and van Wijnen AJ: The osteogenic transcription factor Runx2 regulates components of the fibroblast growth factor/proteoglycan signaling axis in osteoblasts. J Cell Biochem 107: 144-154, 2009.

20. Ngueguim FT, Khan MP, Donfack JH, Siddiqui JA, Tewari D, Nagar GK, Tiwari SC, Theophile D, Maurya R and Chattopadhyay N: Evaluation of cameroonian plants towards experimental bone regeneration. J Ethnopharmacol 141: 331-337, 2012. 